\title{
New high-temperature ICP®-vibration sensors with overload protection to improve measurement uncertainty and reduce measurement errors.
}

\author{
Stefan Meyer \\ PCB Synotech $\mathrm{GmbH}$ \\ Porschestrasse 20-30 \\ 41836 Hückelhoven
}

\begin{abstract}
$I C P \circledast$ technology is an integral part of electronic measuring technology combined with sensors. We find this two-wire technology in laboratories, test benches, in production, in monitoring systems and mobile test installations. Even if it is a simple and proven technique, you should pay attention to some parameters in the sensor selection. New developments provide additional protection against unwanted overloading and also offer higher temperature stability, which improves the values of the measurement uncertainty by a factor of 10.

In the lecture the problem of overloading will be discussed. With examples from practice regarding the reasons for a overloading, detection and prevention. Various application examples are shown. The use of newer generation piezoelectric materials such as the UHT-12 TM industrial single crystal significantly improves the measurement uncertainty and quality of the measurement signals. The technical characteristics of this material are explained and the advantages of using it as a measuring element are shown.
\end{abstract}

\section{Key Words}

ICP $\circledast$, UHT-12 TM, Low Temperature Coefficient (LTC), Ferroelectric Ceramics, Acceleration Sensors, Overload, Low Pass Filter,

\section{The requirements for $I C P \circledast$ Vibration Sensors changed.}

The ICP®-Technique was originally developed for modal- and structural analysis and was also used in vibration machine monitoring as well as applications in research and development. Here, more and more often the limits in temperature and frequency bandwidth were reached. The results are faulty measurement data or defective sensors.

Particular attention must be paid to the materials used for use in space application in order to minimize the outgassing of materials in a vacuum. In addition, there has been a growing interest in being able to use ICP® Vibration sensors even at higher temperatures.

More than 40 years ago, when the ICP® piezoelectric sensor technology was introduced by Piezotronics, Inc., the upper temperature limit was $121^{\circ} \mathrm{C}$ for a long time. This changed with the development of ICP $®$ models with special hightemperature amplifier electronics that could be used up to $163^{\circ} \mathrm{C}$. Continuous development allowed $\mathrm{PCB} \otimes$ to expand its range of applications and improve precision. Current state of the art is the achievement of a continuous operating temperature for ICP $®$ sensors of $+180{ }^{\circ} \mathrm{C}$ with the three-axis measuring models HT356B01 and TLD339A37.

$I C P ®$ is a technology with many variations. Looking at the circuit diagram of an ICP sensor, two components are visible (Figure 1). This is the piezoelectric measuring element and an amplifier or impedance converter. In the sensor part we have the possibility of mechanical design and selection of the piezoelectric material. The electronics offer the possibility of high integration and implementation of additional functions such

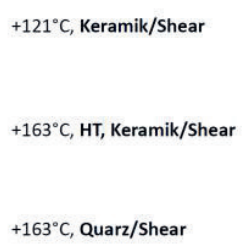

$+163^{\circ} \mathrm{C}, \mathrm{UHT}-12 /$ Shear $\pm 0,009 \% /{ }^{\circ} \mathrm{C}$
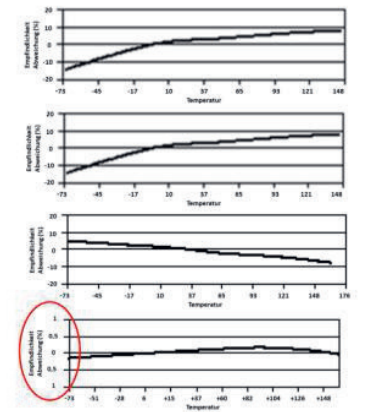
as filters, amplification and automatic parameterization (TEDS).

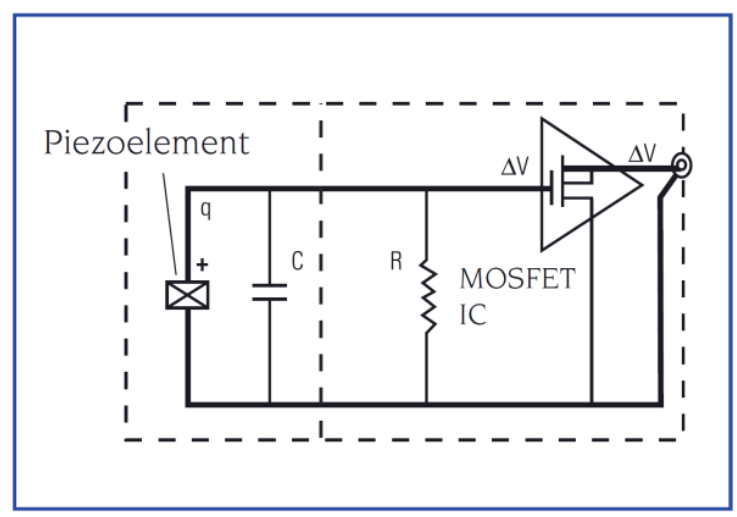

Figure 1: ICP®-Technology (IEPE)

The piezoelectric material is the cause of high measurement uncertainty.

The selection of the piezoelectric material depends on the size, the measuring range, the temperature range and the desired measurement uncertainty. As materials are available versions of the family of ferroelectric ceramics, quartz or industrial single crystals (UHT-12). In recent vibration sensor developments, single crystals such as UHT-12 are used, which have extremely good stability with larger temperature changes. This is an important feature when used in a climate chamber, engine test benches or gas turbines. If we compare the trend of sensitivity over temperature for equivalent sensors with different piezoelectric materials, we can see considerable differences (Figure 2).

Figure 2: Comparison: Temperature coefficients / sensitivity changes over temperature. Comparable vibration sensors with $10 \mathrm{mV} / \mathrm{g}$.

The range of measurement uncertainty has a bandwidth of 1.5 to $25 \%$ and more. The best results are achieved by the sensors with the UHT-12 single crystal. There is now a whole product family of uniaxial and triaxial vibration sensors available with this piezoelectric material, even in different sizes (Figure 3).

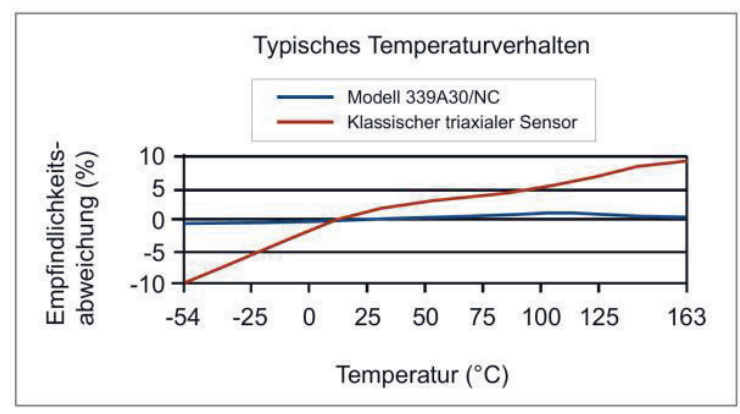

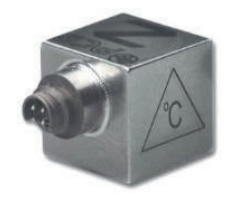

Figure 3: Temperature responce of the sensitivity: standard vs. UHT-12 (339A30 triaxial).

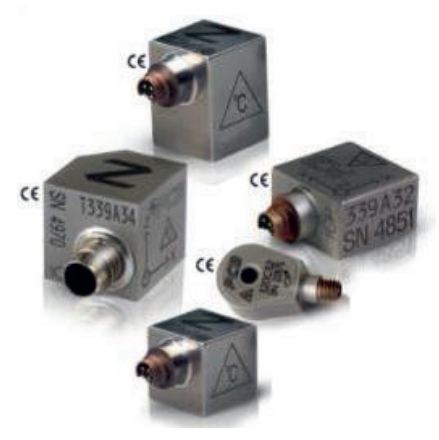

Figure 4: Series 339 products

The relationship between operating temperature and sensitivity is called the temperature coefficient of sensitivity. Since the relationship between temperature and sensitivity is not linear, model-specific characteristics are shown on the data sheets of the sensors and in this brochure, which show the course over the entire temperature range. To minimize the temperature error, sensors with low temperature coefficients should be used, such as the M320C52 and 355M102 uniaxial models, and the 339 series triaxial types equipped with UHT-12 ${ }^{\mathrm{TM}}$ sensor elements.

\section{What is so special about UTH-12?}

The piezoelectric synthetic single crystal (UHT$12^{\mathrm{TM}}$ ) has been developed for high temperature applications. It is very temperature stable and can be reliably used up to $650^{\circ} \mathrm{C}$. The piezoelectric material is not pyroelectric and has a high internal resistance, even at high temperatures. The piezoelectric coefficient $(\mathrm{pC} / \mathrm{N})$ of UHT-12 TM is about three times larger than that of quartz. It can therefore also be used in sensors with ICPtechnology. Vibration sensors with such elements have an extremely low temperature coefficient (Series 339).

\section{Examples of $+180^{\circ} \mathrm{C} \mathrm{ICP} \circledast$ - Accelerometers}

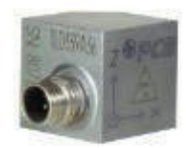


Triaxial TLD339A37, 100mV/g, LP-Filter, TEDS

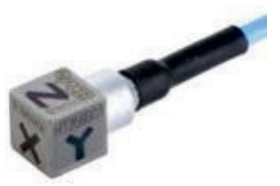

Miniatur Triaxial HT356B01

\section{What's next in the high temperature range of ICP $尺$ sensors?}

The $+180^{\circ} \mathrm{C}$ are currently state of the art and are limited by the electronics. The further development of the electronics (ASIC) brings us further miniaturization with integrated temperature compensation. As a result, even very small sensors with ceramic elements can be considerably improved with regard to measuring inaccuracy. The first candidate with this technique is available with a weight of 0.9 grams, $10 \mathrm{mV} / \mathrm{g},+163^{\circ} \mathrm{C}$, TEDS and temperature response of $\leq 3 \%$ (Figure 5).

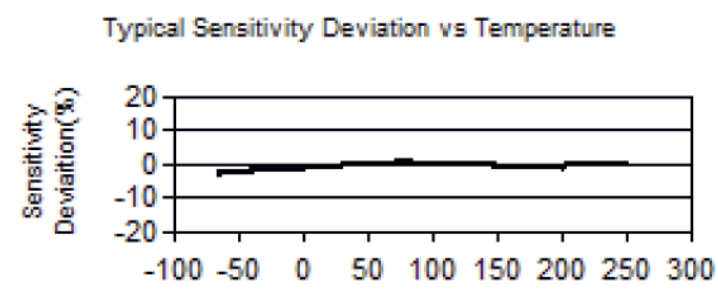

Figure 5: Temperature response Model 352A59

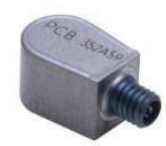

Figure 6: Sub-miniature model 352A59 with advanced electronics

\section{Unusual measurement results with ICP® vi- bration sensors}

After completing a test and evaluating data, test engineers may observe obvious signs of problems within the data that was collected. Many factors can affect the data from a PE accelerometer including measurement range, the measurand input amplitude, the measurand input frequency content, and the data acquisition sample rate. For example, input amplitude levels that are greater than the sensor's measurement range will saturate the amplifier. Input frequency content at or near the sensor's resonant frequency may also saturate the amplifier. The high Qfactor at resonance will cause the sensor to enter an overload recovery state and no meaningful data can be acquired (even with post-process filtering in your DAQ).

\section{Saturation of the amplifier by overload}

Amplitudes greater than the measurement range can saturate the $I C P \circledast$ amplifier in the sensor. Frequency components in the signal, which are in the range of the resonant frequency of the sensor, can also lead to the saturation of the amplifier. The resonance effects in the sensor element mean that the sensor can't capture meaningful data. Filtering afterwards does not lead to useful measurement results.

Typical for overload conditions of the amplifier are exponential decay curves (Figure 7).

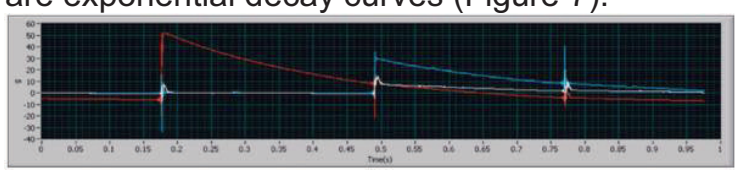

Figure 7: Saturated amplifier

\section{Saturation of the amplifier due to resonance effects}

In some applications, the sensor elements are excited in the region of the resonant frequency. The reason is high-frequency and high-energy pulses, usually caused by metallic shocks, or, more rarely, by high-frequency vibrations with high amplitude, which occur especially in random vibration tests. Input frequencies close to its resonant frequency can cause saturation of the amplifier, generating inaccurate readings. The solution against the problems described is a lowpass filter, between sensor element and amplifier, since a rework of the signal with filter is no longer possible. For applications where such conditions are expected, a filtered model should be selected (Figure 8).

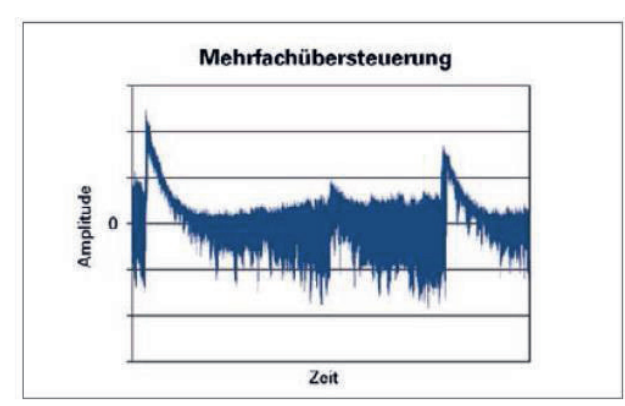


Figure 8: Multiple saturated amplifier

\section{Internal filters help}

Sensors with single or two-pole low pass filters will decrease the chance of amplifier saturation and increase the useable frequency range. Low pass filters will attenuate (suppress) signal generation at or near the resonant frequency of the sensor. This counteracts the gain (high-Q) factor caused by the sensor's mechanical resonance (Figure 9).

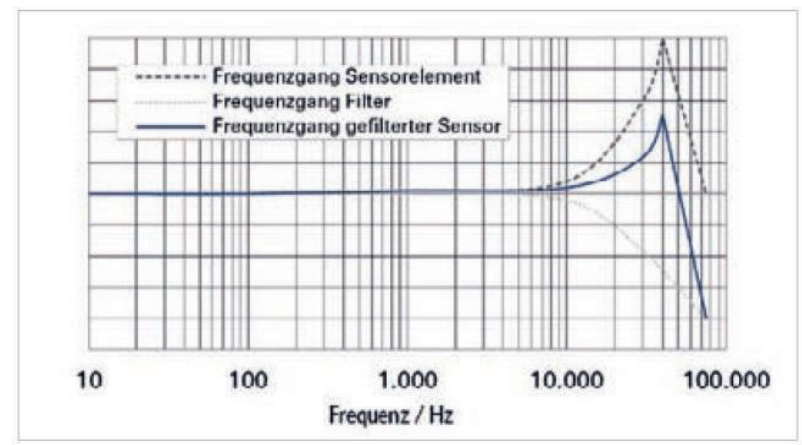

Figure 9: Influence of the filter on the frequency response

\section{Where should sensors with low temperature coefficients and integrated low-pass filter be used.}

- Ideal for component and component testing in climate chambers.

- Measurements on vehicle powertrain.

- HALT/HASS measurements.

Saturated amplifier; real-life examples.

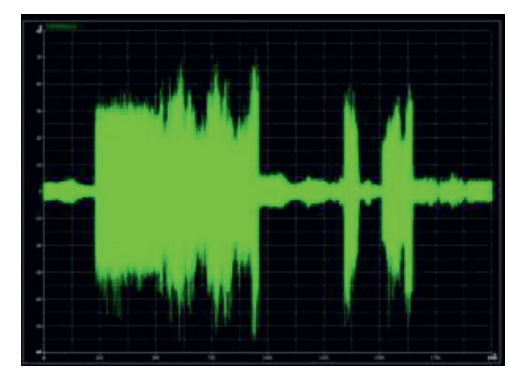

Error image from the aviation industry.

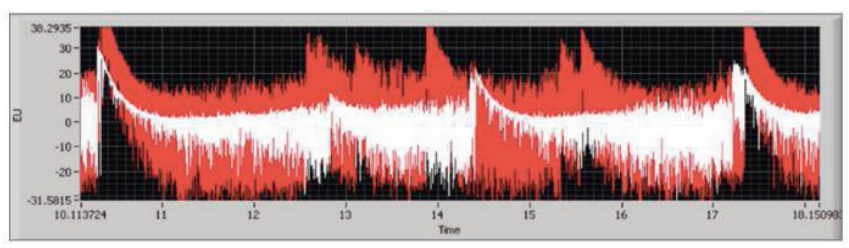

Saturated amplifier by resonance excitation 


\section{Summary}

- By using sensors with a particularly low temperature coefficient, thermal measurement errors can be reduced to a minimum.

- Such transducers use the temperaturestable sensor element material UHT$12^{\mathrm{TM}}$, which has been specially developed for piezoelectric high-temperature sensors.

- It delivers precise measurement results over the entire operating temperature range of -54 to $163^{\circ} \mathrm{C}$, even at changing temperatures.

- Many ICP ${ }^{\circledR}$ high-temperature sensors from the $\mathrm{PCB}^{\circledR}$ portfolio have integrated low-pass filters.

- They are always useful when highfrequency vibrations or high-energy pulses are exposed to the sensor.

- Such impulses are caused by metallic impacts, such as can occur in the combustion events in drive trains.

- The measuring element of an acceleration sensor is excited by such shocks in its resonance, which can cause saturation of the amplifier in unfiltered models.

- A correction of the signal data after a saturation is not possible, not even by filtering, since the raw data has already been recorded incorrectly.

- Filtered sensors eliminate this source of error.

\section{References}

- Technische Information TN-30 „Ungewöhnliche Messergebnisse bei piezoelektrischen Beschleunigungssensoren" (PCB Synotech).

- Technische Information TN-051 „Piezoelektrische Aufnehmer mit eingebauer Mikroelektronik nach dem ICP ${ }^{{ }_{-}}$ Konzept" (PCB Synotech).

- $\quad \mathrm{ICP}{ }^{\circledR}$-HochtemperaturBeschleunigungssensoren (PCB Synotech).

- Sensoren für den Prüfstandsbau (PCB Synotech). 